\title{
Systemic and Urinary Neutrophil Gelatinase-Associated Lipocalins Are Poor Predictors of Acute Kidney Injury in Unselected Critically Ill Patients
}

\author{
Annick A. Royakkers, ${ }^{1,2}$ Catherine S. Bouman, ${ }^{2}$ Pauline M. Stassen, ${ }^{3}$ \\ Joke C. Korevaar, ${ }^{4}$ Jan M. Binnekade, ${ }^{1}$ Willem van de Hoek, ${ }^{5}$ Michael A. Kuiper, ${ }^{6,7}$ \\ Peter E. Spronk, ${ }^{7,8}$ and Marcus J. Schultz ${ }^{2,3,7}$ \\ ${ }^{1}$ Department of Intensive Care Medicine, Tergooi Hospitals, Location Blaricum, 1261 AN Blaricum, The Netherlands \\ ${ }^{2}$ Department of Intensive Care Medicine, Academic Medical Center, University of Amsterdam, 1105 AZ Amsterdam, The Netherlands \\ ${ }^{3}$ Laboratory for Experimental Intensive Care and Anesthesiology $(L \cdot E \cdot I \cdot C \cdot A)$, Academic Medical Center, University of Amsterdam, \\ 1105 AZ Amsterdam, The Netherlands \\ ${ }^{4}$ Department of Clinical Epidemiology and Biostatistics, Academic Medical Center, University of Amsterdam, \\ 1105 AZ Amsterdam, The Netherlands \\ ${ }^{5}$ Department of Intensive Care Medicine, Scheper Ziekenhuis Emmen, 7824 AA Emmen, The Netherlands \\ ${ }^{6}$ Department of Intensive Care Medicine, Medical Center Leeuwarden, 8934 AD Leeuwarden, The Netherlands \\ ${ }^{7}$ HERMES Critical Care Group, 1105 AZ Amsterdam, The Netherlands \\ ${ }^{8}$ Department of Intensive Care Medicine, Gelre Hospitals, Location Lucas, 7334 DZ Apeldoorn, The Netherlands
}

Correspondence should be addressed to Annick A. Royakkers, aro@hetnet.nl

Received 11 June 2012; Accepted 19 September 2012

Academic Editor: Manuel E. Herrera-Gutiérrez

Copyright ( 2012 Annick A. Royakkers et al. This is an open access article distributed under the Creative Commons Attribution License, which permits unrestricted use, distribution, and reproduction in any medium, provided the original work is properly cited.

\begin{abstract}
Background. Neutrophil gelatinase-associated lipocalin (NGAL) in serum and urine have been suggested as potential early predictive biological markers of acute kidney injury (AKI) in selected critically ill patients. Methods. We performed a secondary analysis of a multicenter prospective observational cohort study of unselected critically ill patients. Results. The analysis included 140 patients, including 57 patients who did not develop AKI, 31 patients who developed AKI, and 52 patients with AKI on admission to the ICU. Levels of sNGAL and uNGAL on non-AKI days were significantly lower compared to levels of sNGAL on RIFLE $_{\text {RISK }}$ days, RIFLE INJURY days, and RIFLE interval (CI) 0.27-0.63) and 0.53 (CI 0.38-0.67), 2 days and 1 day before development of AKI, respectively. The AUC of uNGAL for predicting AKI was also low: 0.48 (CI 0.33-0.62) and 0.48 (CI 0.33-0.62), 2 days and 1 day before development of AKI, respectively. AUC of sNGAL and uNGAL for the prediction of renal replacement therapy requirement was 0.47 (CI $0.37-0.58)$ and 0.26 (CI 0.03-0.50). Conclusions. In unselected critically ill patients, sNGAL and uNGAL are poor predictors of AKI or RRT.
\end{abstract}

\section{Introduction}

Acute kidney injury (AKI) represents a frequent complication in critically ill patients, with high rates of morbidity and mortality [1-5]. AKI requiring renal replacement therapy (RRT) occurs in up to $5 \%$ of patients with AKI, in whom the mortality rate approaches $80 \%$ [4]. The lack of early biological markers of renal injury prevents timely patient management decisions, including withholding nephrotoxic agents, administration of putative therapeutic agents, and the initiation of RRT.

Recently, neutrophil gelatinase-associated lipocalin (NGAL) has been implicated as an early predictive biological marker of renal injury [6]. NGAL is a ubiquitous $25 \mathrm{kDa}$ protein, covalently bound to gelatinase from human neutrophils, which is normally expressed in very low concentrations in several human tissues, including the kidney $[7,8]$. Expression of NGAL increases in the presence of inflammation 
and injured epithelia, including the kidney after ischemia reperfusion injury and nephrotoxicity $[7,8]$. Systemic NGAL appeared to be of diagnostic as well as prognostic value for AKI in previous studies of critically ill patients [9]. However, the role of NGAL in critically ill patients has mainly been studied in highly selected populations, including children and adults after cardiac surgery $[10,11]$ or after intravenous administration of contrast [12]. In these populations AKI etiology is clear and timing of the insult is often precisely known.

In the present study we evaluated the performance of NGAL in a group of unselected critically ill intensive care unit (ICU) patients, in which AKI etiology and timing are most of the time unclear. Thus, we chose to study a population reflecting daily practice in our centers and tested two hypotheses. First, we hypothesized that NGAL in serum (sNGAL) and urine (uNGAL) can predict AKI 1 to 2 days earlier than the RIFLE criteria in patients who develop AKI after admission to the ICU. Second, we hypothesized that sNGAL and UNGAL predict the need for RRT, in unselected ICU patients.

\section{Methods}

2.1. Study Design. This study is a secondary analysis of a multicenter prospective observational cohort study of unselected critically ill patients in 5 multidisciplinary, closedformat ICUs, in which we collected serial serum and urine samples and determined the first day of AKI based on the RIFLE (risk, injury, failure, loss, and end-stage renal disease) classification system $[13,14]$. The institutional review board of all participating institutions approved the protocol, and written informed consent was obtained from all patients or next of kin.

2.2. Patients. Patients who were older than 18 years, with an expected duration of mechanical ventilation of at least 48 hours and/or an expected length of ICU stay of at least 72 hours, were enrolled within 48 hours of ICU admission. Chronic RRT was an exclusion criterion.

2.3. Data Collected. Demographic data, admission diagnosis, reasons to initiate RRT (oliguria or anuria, high sCr/high sUr, or acidosis), acute physiology age and chronic health evaluation (APACHE) II scores and simplified acute physiology scores (SAPS) II $[15,16]$ were documented in the first 24 hours after admission. Routine laboratory data, including plasma creatinine, were measured daily.

2.4. Baseline Renal Function and Definition of AKI. In order to define the baseline renal function we compared the premorbid levels of serum creatinine $(\mathrm{sCr})$ within 1 year prior to ICU admission with the sCr at ICU admission. The lower of these 2 values served as baseline renal function. In case a premorbid sCr was unavailable, baseline renal function was estimated by solving the modification of diet in renal disease (mdrd) equation, with the assumption of a near lower limit of normal glomerular filtration rate (GFR) of $75 \mathrm{~mL} / \mathrm{min} / 1.73 \mathrm{~m}^{2}[17]$.
The presence of AKI on admission and development of AKI during stay in ICU were scored using the creatinine and urine output criteria of the RIFLE classification system for AKI [13]. In an additional analysis development of AKI during stay in ICU was scored using solely the creatinine criteria. The first day of AKI (the first RIFLE event) was termed AKI day 0; the 2 days prior to this day were termed AKI day 1 and AKI day 2, respectively.

2.5. Sampling and Measurement of NGAL. Blood and urine sampling for NGAL measurements was performed on days 0,1 , and alternate days until the start of RRT, ICU discharge, or death, whatever came first. Blood samples were drawn into sterile Vacutainer tubes and centrifuged at $1.500 \times \mathrm{g}$ for 10 minutes at $4^{\circ} \mathrm{C}$. The supernatants were stored at $-80^{\circ} \mathrm{C}$. Urine samples were taken from a 3-hour urine collection period after assessment of the urine volume, centrifuged, and also stored at $-80^{\circ} \mathrm{C}$.

All samples of NGAL were measured batch wise by means of a commercial ELISA (R\&D Systems, Abingdon, UK) according to manufactures recommendation. We tested multiple dilutions on the samples and made them in duplicate. To compensate for differences in urine flow rate, we normalized the urinary excretion of NGAL for moles of urinary creatinine ( $\mathrm{uNGAL}_{\text {corr. }}$ ) [18]. In addition, as proposed before [19], we used the ratio of sNGAL to UNGAL in additional analyses.

2.6. Effect Size. To find out what sort of effect size was to be expected in this secondary analysis, we calculated the standard error, 0.061, using the actual number of included patients in our study. The accompanying 95\% confidence limits were 0.63 to 0.87 given the estimated minimal clinically relevant area under the curve to be at least 0.70 .

2.7. Statistical Analysis. The first RIFLE event (risk, injury, or failure, using the combination of creatinine and urine output criteria, or the creatinine criterion alone) served as the primary endpoint. Initiation of RRT served as a secondary endpoint. Data were analyzed using the Statistical Package for the Social Sciences (SPSS) for Windows, version 17.0 (SPSS, Chicago IL, USA).

Continuous variables were expressed as mean $( \pm \mathrm{SD})$ or median with (IQR). Categorical variables were expressed as counts and percentages. Normally distributed variables were compared using one-way analysis of variance with Bonferroni's correction for multiple comparisons. For significant findings, post hoc $t$-test was applied. Kruskall-Wallis oneway analysis of variance was used to compare nonnormally distributed variables. Chi-square testing was used to test frequencies between groups. Linear mixed models were used to compare NGAL levels among RIFLE stages. Testing was two-tailed; $P<0.05$ was considered statistically significant.

Patients with AKI at admission were excluded from the diagnostic analysis and included only in the prognostic analysis. We used day 2 and day 1 in patients who developed AKI with day 0 and day 1 in patients who never developed AKI. This time period was chosen as patients who developed AKI fulfilled RIFLE criteria after 2 (1-2) days. 
TABLE 1: Group characteristics.

\begin{tabular}{|c|c|c|c|c|}
\hline & $\begin{array}{c}\text { Patients who never } \\
\text { developed AKI } \\
(N=57)\end{array}$ & $\begin{array}{c}\text { Patients who developed } \\
\text { AKI } \\
(N=31) \\
\end{array}$ & $\begin{array}{c}\text { Patients with AKI on } \\
\text { admission } \\
(N=52)\end{array}$ & $P$ value \\
\hline Age (years) & $58.8(16.1)$ & $67.1(15.8)$ & $74.4(9.4)$ & $<0.05$ \\
\hline Gender (male) (\%) & $37(64.9 \%)$ & $21(67.7 \%)$ & $32(61.5)$ & 0.84 \\
\hline Weight (kg) & $75.7(14.7)$ & $81.6(14.9)$ & $81.1(19.7)$ & 0.17 \\
\hline Height $(\mathrm{cm})$ & $176.0(8.4)$ & $175.2(8.5)$ & $171.2(17.1)$ & 0.12 \\
\hline APACHE II score & $18.5(9.4)$ & $19.3(8.3)$ & $23(11.5)$ & 0.06 \\
\hline SAPS II & $35.9(12)$ & $42.8(15)$ & $47.1(14.8)$ & $<0.05$ \\
\hline RIFLE baseline $\mathrm{s} C \mathrm{r}(\mu \mathrm{mol} / \mathrm{L})$ & $69.9(18.7)$ & $77.9(15.9)$ & $76.3(19.8)$ & 0.16 \\
\hline sUrea $(\mathrm{mmol} / \mathrm{L})$ & $7.9(5.9-11.2)$ & $11.7(8.1-17.2)$ & $12.4(8.1-22.4)$ & $<0.05$ \\
\hline $\mathrm{sCr}(\mu \mathrm{mol} / \mathrm{L})$ & $62(50-78)$ & $86(72-104)$ & $110(73-177)$ & $<0.05$ \\
\hline sNGAL (ng/mL) & $269(180-398)$ & $307(187-460)$ & $343(238-652)$ & $<0.05$ \\
\hline uNGAL (ng/mL) & $99(41-301)$ & $149(52-405)$ & $289(92-602)$ & $<0.05$ \\
\hline $\mathrm{uNGAL}_{\text {corr }}(\mathrm{mg} / \mathrm{molCr})$ & $23(9-64)$ & $40(13-142)$ & $23(8-101)$ & 0.05 \\
\hline \multicolumn{5}{|l|}{ Primary diagnosis $(n)(\%)$} \\
\hline $\mathrm{CPB}$ & $1(1.7 \%)$ & $2(6.4 \%)$ & - & 0.25 \\
\hline Cardiovascular failure & $1(1.7 \%)$ & $4(12.9 \%)$ & $4(7.7 \%)$ & 0.11 \\
\hline Cerebrovascular event & $2(3.5 \%)$ & $0(0 \%)$ & $0(0 \%)$ & - \\
\hline Hemorrhagic shock & $7(12.3 \%)$ & $1(3.2 \%)$ & $5(9.6 \%)$ & 0.37 \\
\hline Multiple trauma & $3(5.3 \%)$ & $2(6.4 \%)$ & $1(1.9 \%)$ & 0.55 \\
\hline Elective major surgery & $1(1.8 \%)$ & $1(3.2 \%)$ & $5(9.6 \%)$ & 0.15 \\
\hline Respiratory failure & $22(38.6 \%)$ & $10(32.2 \%)$ & $14(26.9 \%)$ & 0.43 \\
\hline Septic shock & $20(35.1 \%)$ & $11(35.5 \%)$ & $24(46.2 \%)$ & 0.44 \\
\hline \multicolumn{5}{|l|}{ Admission category $(n)(\%)$} \\
\hline Medical & $27(47.4 \%)$ & $14(45.2 \%)$ & $21(40.4 \%)$ & 0.76 \\
\hline Surgical & $30(52.6 \%)$ & $17(54.8 \%)$ & $31(59.6 \%)$ & 0.76 \\
\hline $\begin{array}{l}\text { Days from admission until AKI } \\
\text { (median and range) }\end{array}$ & NA & $2(1-2)$ & 0 & - \\
\hline \multicolumn{5}{|l|}{ Worst AKI score in ICU $(n)(\%)$} \\
\hline Risk & - & $25(80 \%)$ & $22(42 \%)$ & $<0.05$ \\
\hline Injury & - & $4(12 \%)$ & $13(25 \%)$ & 0.19 \\
\hline Failure & - & $2(6 \%)$ & $17(32 \%)$ & 0.006 \\
\hline $\mathrm{CVVH}$ & $1(2 \%)$ & $3(10 \%)$ & $8(15 \%)$ & $<0.05$ \\
\hline LOS (days) & $5(3-8)$ & $8(5-18)$ & $6(3-9)$ & 0.001 \\
\hline ICU mortality & 0 & $4(13 \%)$ & $8(15 \%)$ & $<0.05$ \\
\hline Hospital mortality & $4(7.0 \%)$ & $5(16.1 \%)$ & $15(29 \%)$ & $<0.05$ \\
\hline
\end{tabular}

We assessed sNGAL and UNGAL on their ability to detect AKI or predict need for RRT by calculation of the area under the curve (AUC) of the receiver-operating characteristic (ROC) plot.

\section{Results}

3.1. Patients. The original study included 170 patients; 19 patients were excluded because data collection was incomplete. Of the remaining 151 patients, samples were no longer available for analysis of NGAL levels in 11 patients: 3 patients who did not develop AKI, 4 patients who developed AKI, and 4 patients with AKI on admission. Therefore, the final analysis included 140 patients, of whom 57 patients did not develop AKI, 31 patients developed AKI, and 52 patients with AKI on admission, when using the MDRD-based baseline $\mathrm{sCr}$ and classifying patients according to the creatinine and urine output criteria of RIFLE. There were no differences in baseline characteristics between the original 170 patients included in the study and 140 patients finally analyzed here.

Baseline demographic data are presented in Table 1. Renal and outcome data are presented in Table 2. Patients who developed AKI were significantly older compared to patients who did not develop AKI. There were no significant differences among the groups in terms of premorbid hypertension, diabetes, or chronic kidney disease. The MDRD 
TABLE 2: Characteristics of acute kidney injury patients by presence or absence of CVVH.

\begin{tabular}{|c|c|c|c|}
\hline & $\begin{array}{c}\text { CVVH } \\
(N=11)\end{array}$ & $\begin{array}{c}\text { no CVVH } \\
(N=72)\end{array}$ & $P$ value \\
\hline Age (years) & $68.9(11.6)$ & $72.1(12.7)$ & 0.43 \\
\hline Gender (male) & $7(63.6 \%)$ & $46(63.9 \%)$ & 0.99 \\
\hline APACHE II score & $28(20-30)$ & $19(14-25)$ & 0.05 \\
\hline SAPS II & $51(44-57)$ & $45(35-51)$ & 0.11 \\
\hline sUrea $(\mathrm{mmol} / \mathrm{L})$ & $11(7-23)$ & $10(7-18)$ & 0.24 \\
\hline $\mathrm{sCr}(\mu \mathrm{mol} / \mathrm{L})$ & $147(94-299)$ & $100(76-139)$ & 0.03 \\
\hline sNGAL (ng/mL) & $338(251-798)$ & $341(205.4-622)$ & 0.56 \\
\hline uNGAL (ng/L) & $303(39-750)$ & $219(92-535)$ & 0.90 \\
\hline $\mathrm{uNGAL}_{\text {corr. }}(\mathrm{mg} / \mathrm{mol} \mathrm{Cr})$ & $38(7-342)$ & $34(9-114)$ & 0.51 \\
\hline Urine output (mL/day) & $1480(493-1960)$ & $2535(1826-3792)$ & $<0.001$ \\
\hline AKI prior to CVVH (days) & $1(0-4)$ & NA & - \\
\hline
\end{tabular}

TABle 3: AUC (CI) for sNGAL, uNGAL, and uNGAL $_{\text {corr. }}$ in predicting AKI.

\begin{tabular}{lcc}
\hline & Day 2 & Day 1 \\
\hline sNGAL & $0.45(0.27$ to 0.63$)$ & $0.53(0.38$ to 0.67$)$ \\
uNGAL & $0.48(0.33$ to 0.62$)$ & $0.48(0.33$ to 0.62$)$ \\
uNGAL $_{\text {corr. }}$ & $0.47(0.29$ to 0.66$)$ & $0.65(0.51$ to 0.79$)$ \\
sNGAL/uNGAL & $0.60(0.41$ to 0.80$)$ & $0.47(0.31$ to 0.63$)$ \\
\hline
\end{tabular}

based baseline sCr was used in $10 \%$ of patients who never developed AKI, 13\% of patients who developed AKI, and $29 \%$ of patients with AKI on admission.

Patients who developed AKI fulfilled RIFLE criteria after 2 (1-2) days. Continuous venovenous hemofiltration (CVVH) was started in 11 out of 83 patients who presented with or developed AKI (13\%). The reason to initiate RRT was oliguria/anuria in 7 patients, acidosis in 1 patient, and high sCr or high sUr in 3 patients. The duration between AKI day 0 and start of RRT was median $1(0-4)$ day. In comparison with the non-RRT patient, AKI patients who required RRT had significantly higher APACHE II scores on admission and produced less urine.

3.2. Serum and Urine Levels of NGAL. Figure 1 shows levels of sNGAL and uNGAL. Compared to levels of sNGAL on non-AKI days, significantly higher levels of sNGAL were found on RIFLE RISK $_{\text {days, RIFLE }}$ INJURY days and RIFLEFAILURE days. Serum NGAL levels were not significantly different among the 3 RIFLE categories.

Similarly, compared to levels of uNGAL on non-AKI days, significantly higher levels of UNGAL were found on RIFLE RISK $_{\text {days, RIFLE }}$ INJURY days, and RIFLE FAILURE days. Levels of uNGAL were also not significantly different among the 3 RIFLE categories except for RIFLE RISK versus RIFLEFAILURE. Differences in levels of uNGAL per RIFLE category remained similar when urine NGAL was corrected for moles of urinary creatinine.

3.3. Prediction of AKI. We compared levels of sNGAL and uNGAL in the 2 days prior to AKI from patients who
TABle 4: AUC (CI) for sNGAL, uNGAL, and $\mathrm{uNGAL}_{\text {corr. }}$ in predicting RRT.

\begin{tabular}{lc}
\hline sNGAL & $0.47(0.37$ to 0.58$)$ \\
uNGAL & $0.26(0.03$ to 0.50$)$ \\
uNGAL $_{\text {corr. }}$ & $0.27(0.0$ to 0.57$)$ \\
sNGAL/uNGAL & $0.26(0.01$ to 0.51$)$ \\
\hline
\end{tabular}

developed AKI, with the first 2 study days of admission in patients who did not develop AKI (Figure 2). The areas under the ROC curve of sNGAL and UNGAL for predicting AKI were low and only slightly improved by normalizing the excretion of NGAL for moles of urinary creatinine (Table 3 ). Similarly, using the ratio of sNGAL to uNGAL the AUC only slightly improved (Table 3 ).

When we did not use the MDRD-based baseline sCr results were not different. Also, when we only used the creatinine criterion of RIFLE to classify the presence of AKI, and non-AKI and AKI days, areas under the ROC curve of sNGAL and UNGAL for predicting AKI remained low (see electronic supplement supplementary material available online at doi:10.1155/2012/712695).

3.4. Prediction of RRT. The areas under the ROC curve of sNGAL and UNGAL for predicting RRT requirement were also low, and did not improve by normalizing the excretion of NGAL for moles of urinary creatinine or when using the ratio of sNGAL to uNGAL (Table 4).

\section{Discussion}

The aims of this prospective multicenter study were to evaluate whether NGAL in serum and urine can detect AKI earlier than the RIFLE criteria in unselected critically ill patients, and whether NGAL in serum and urine can predict RRT requirement. Levels of sNGAL and UNGAL on non-AKI days were significantly lower compared with that on RIFLERISK-INJURY-FAILURE days. Levels of sNGAL and uNGAL were not different between the patients who finally proceeded with RRT and patients who did not need RRT. 


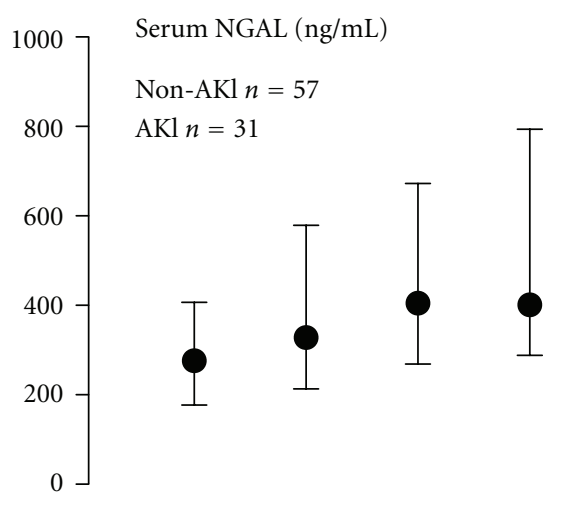

None Risk Injury Failure

(a)

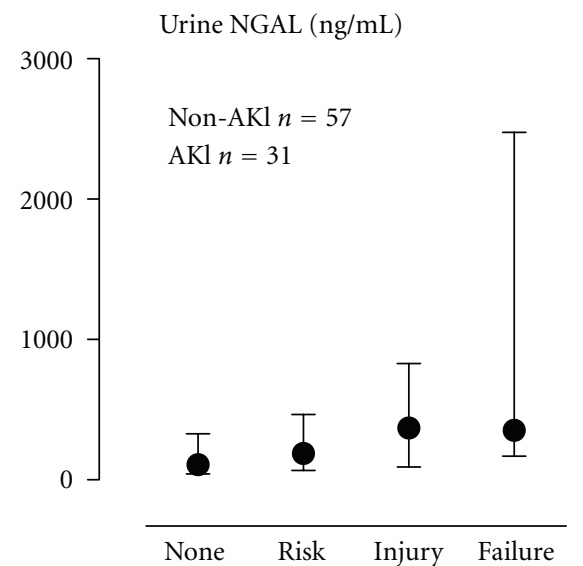

(b)

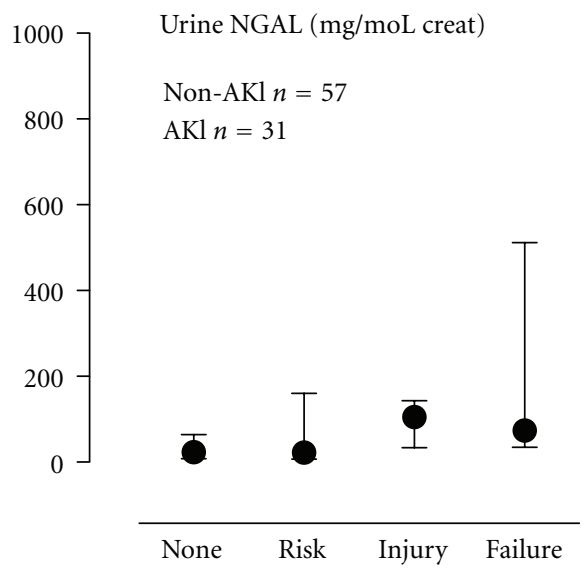

(c)

FIGURE 1: Neutrophil gelatinase-associated lipocalin (NGAL) in serum and urine per RIFLE severity. On nonacute kidney injury (AKI) days NGAL in serum and urine was lower $(P<0.05)$ compared with days fulfilling the RIFLE ${ }_{\text {RISK }}$, RIFLE INJURY, and RIFLE $_{\text {FAILURE }}$ criteria. Non-AKI patients, $N=57$; AKI patients $N=31$.

The predictive ability of sNGAL and UNGAL was poor, both for detecting AKI and for predicting need for RRT.
Our study is amongst the first studies investigating whether NGAL predicts the development of AKI in unselected critically ill ICU patients in which AKI etiology and timing are often unclear. Our study knows several limitations, though. First, our sample size was relatively small. Second, the MDRD-based baseline sCr had to be used in 18\% of our patients. Missing preadmission sCr value is a recognized problem in AKI research, which may lead to misclassification of the incidence of AKI [20]. Contrary, the use of surrogate measures for baseline renal function prevents selection bias. Using MDRD-based baseline, sCr gave us the opportunity to analyze all patients, which otherwise was not possible. Notably, when we did not use the MDRD-based baseline sCr results were not different. Third, the definition of a disease or clinical entity is critical in biomarker research, and the RIFLE criteria may not be an adequate "gold standard" for AKI. The consensus RIFLE definition for AKI is based on sCr and/or urine output. These are functional parameters and may not be appropriate for the detection of injury to the kidney.

We were also the first to use the RIFLE classification with both $\mathrm{sCr}$ and urine output, to classify patients. This might have affected our outcomes. Indeed, by using the urine output criterion in addition to the $\mathrm{sCr}$, we may have classified more patients as having AKI. We consider our approach, based upon consensus, more appropriate. Furthermore, when we only used the creatinine criterion of RIFLE, the predictive ability of sNGAL and UNGAL remained poor.

Our results are in contrast with those from other investigations. Indeed, studies investigating sNGAL and/or uNGAL for the prediction of AKI in patients after cardiac surgery [ 10 , 11, 21-27], patients with multiple trauma [28], and patients with sepsis or SIRS [29, 30] showed excellent performances for NGAL as a predictive biological marker of AKI, with areas under the ROC curve of sNGAL and up to 0.91 and of uNGAL up to 0.99 . This discrepancy may not come as a surprise, since in these patient groups usually the direct cause of AKI as well as its timing is often obvious. In addition, since collection of specimens in our study could start the next day of admission and specimens were collected on alternate days after the first 2 days, we may have missed peak NGAL levels in our study. However, our approach better reflects daily practice, which is most of the time very different from the ideal research setting.

It should be noted, though, that the results of our study are also different from results from 3 recently published studies that focused on the diagnostic performance of serum NGAL in a more heterogeneous ICU populations [31-33]. Opposite to our findings, these 3 studies suggested sNGAL to be a good and early biological marker of AKI, with increased levels of sNGAL 48 hours before RIFLE criteria were met, and even on admission. Cruz et al. studied 301 consecutive patients admitted to a general medical-surgical ICU [31]. The primary outcome was AKI, defined as an increase in plasma creatinine of at least $50 \%$ from baseline or a reduction in urine output to $<0.5 \mathrm{~mL} / \mathrm{kg} /$ hour for $>6$ hours. sNGAL was a good diagnostic marker for AKI development within the next 48 hours (area under the ROC curve 0.78 (CI 0.65-0.90) and for RRT requirement (area under the 
Serum creat $(\mu \mathrm{mol} / \mathrm{L})$

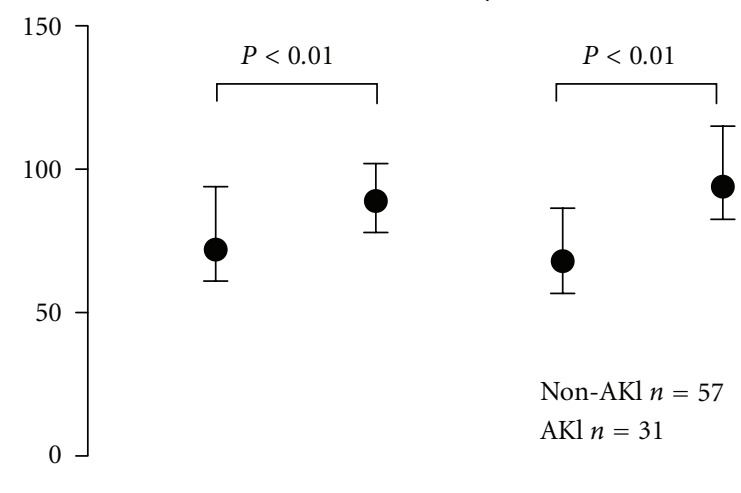

ICU day $0 \quad$ AKl day-2 $\quad$ ICU day $1 \quad$ AKl day-1

(a)

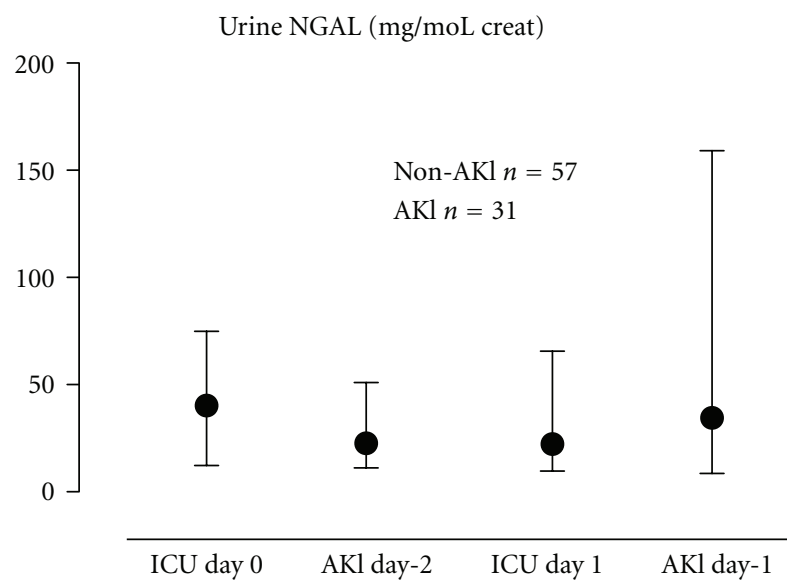

(c)



$\begin{array}{llll}\text { ICU day } 0 & \text { AKl day-2 } & \text { ICU day } 1 \quad \text { AKl day-1 }\end{array}$

(b)

Urine NGAL (ng/mL)

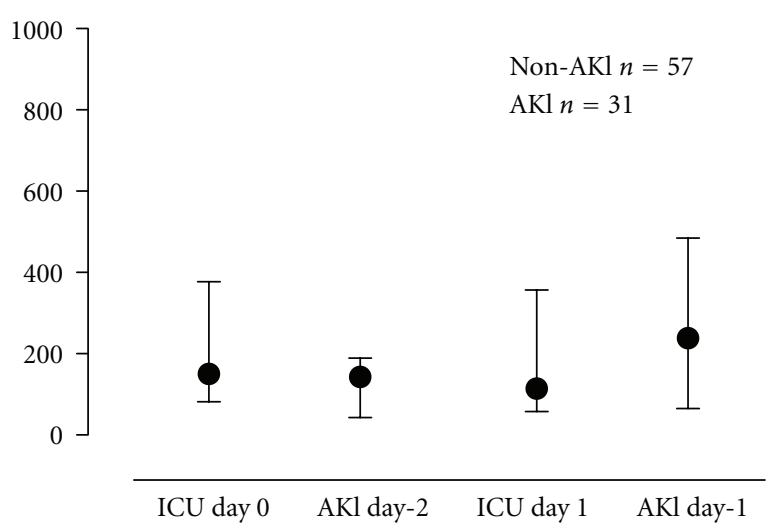

(d)

FIgURE 2: Neutrophil gelatinase-associated lipocalin (NGAL) in serum and urine and creatinine 1 and 2 days prior to acute kidney injury (AKI). Levels are compared to day 0 and day 1 in patients who never developed AKI. Non-AKI patients, $N=57 ; \mathrm{AKI}$ patients, $N=31$.

ROC curve 0.82 (CI 0.70-0.95). Constantin et al. studied 88 critically ill patients [32]. Focusing on patients without AKI on admission, the area under the ROC curve of sNGAL was 0.96 (CI 0.86-0.99) for prediction of AKI. de Geus et al. studied the predictive value of NGAL in 632 critically ill patients [33]. In this study urine was collected from admission up to 72 hours after admission. The AUC for sNGAL in predicting AKI was $0.77 \pm 0.05$ (RIFLE $0.80 \pm 0.06\left(\right.$ RIFLE $\left._{\text {INJURY }}\right)$, and $0.86 \pm 0.06$ (RIFLEE FAILURE); the AUC for UNGAL in predicting AKI was $0.80 \pm 0.04$ (RIFLE RISK $), 0.85 \pm 0.04$ (RIFLE INJURY), and $0.88 \pm 0.04$ (RIFLE FAILURE . Several differences in study designs may explain the opposite results. First, Constantin et al. used only one single measurement of uNGAL on admission, since their primary endpoint was the value of NGAL to predict AKI on admission to the ICU [32]. In the studies by Cruz et al. and de Geus et al. patients who already had AKI on admission were not excluded, which may have resulted in higher NGAL levels $[31,33]$. Indeed, in the study by Cruz et al. $29 \%$ of all patients (67\% of all AKI patients) had AKI on admission [31] in the study by de Geus et al. 59\% of all patients had AKI on admission [33].
Notably, de Geus et al. found increased uNGAL levels in septic patients without AKI, while levels of sNGAL were not different between patients who developed AKI and those who did not. This is in contrast to a study of adult septic patients by Mårtensson et al. [34]. While in this study the AUC for sNGAL was low (0.67), the AUC for uNGAL was good (0.86).

As described by Haase et al. [9], the use of a standardized NGAL assay reported a better AUC for NGAL than individually developed research-based assays. Cruz et al. and Constantin et al. used the Triage Meter (Biosite, San Diego) $[31,32]$. In our study we used a commercial ELISA. It is uncertain whether this difference explains the differences between our study results and those from other studies.

A large proportion of our patients already had AKI on admission. This is similar to other studies on predictive biomarkers for AKI in a heterogeneous ICU population $[31,33]$. It makes little sense to predict AKI in patients who already have AKI, except if NGAL predicts progression in AKI severity, including the need for RRT. Unfortunately, in our study both sNGAL and UNGAL were no predictors of RRT requirement, although it must be mentioned that the number of patients eventually requiring RRT in our study 
was small. Several factors can be of influence on our results regarding the predictability of need for RRT. For instance, the reason for initiating RRT in our cohort was much more diverse than in patients after cardiopulmonary bypass $[10$, 11, 22-27].

Nearly $40 \%$ of our patients never developed AKI, yet some of these patients had increased levels of NGAL, both in serum and urine. A possible explanation for this finding could be the published reference intervals of sNGAL and uNGAL [35]. They were determined in healthy volunteers with no history of renal disease and may not apply to our critically ill population.

In conclusion, in this multicenter study of unselected critically ill patients, both sNGAL and uNGAL were poor predictive biological markers for AKI. Moreover, sNGAL and uNGAL did not predict the need of RRT.

\section{Authors' Contributions}

A. Royakkers, C. Bouman, J. Korevaar, P. Stassen, and M. Schultz contributed to the conception and design of the study. A. Royakkers, W. van de Hoek, M. Kuiper, P. Stassen, and M. Schultz performed the study. PS performed the NGAL measurements. JK, JMB and CB performed the statistical analysis. AR and CB drafted the paper with the assistance of MJS. All authors read and approved the final paper.

\section{Conflict of Interests}

None of the authors have any conflict of interests to disclose.

\section{Acknowledgments}

The authors thank the medical and nursing staff of the intensive care units of all participating centers for their cooperation and support, particularly J. Hofhuis, M. Koopmans, H. Lu, and W. Chen. They thank A.M. Tuip-de Boer for her help with measuring levels of NGAL. They thank D.H. Koning for developing the database.

\section{References}

[1] E. Andrikos, P. Tseke, O. Balafa et al., "Epidemiology of acute renal failure in ICUs: a multi-center prospective study," Blood Purification, vol. 28, no. 3, pp. 239-244, 2009.

[2] P. Kes and N. B. Jukic, "Acute kidney injury in the intensive care unit," Bosnian Journal of Basic Medical Sciences, vol. 10, supplement 1, pp. S8-12, 2010.

[3] N. V. Kolhe, P. E. Stevens, A. V. Crowe, G. W. Lipkin, and D. A. Harrison, "Case mix, outcome and activity for patients with severe acute kidney injury during the first 24 hours after admission to an adult, general critical care unit: application of predictive models from a secondary analysis of the ICNARC Case Mix Programme Database," Critical Care, vol. 12, supplement 1, p. S2, 2008.

[4] S. Uchino, J. A. Kellum, R. Bellomo et al., "Acute renal failure in critically ill patients: a multinational, multicenter study," Journal of the American Medical Association, vol. 294, no. 7, pp. 813-818, 2005.

[5] W. Y. Park, E. A. Hwang, M. H. Jang, S. B. Park, and H. C. Kim, "The risk factors and outcome of acute kidney injury in the intensive care units," Korean Journal of Internal Medicine, vol. 25, no. 2, pp. 181-187, 2010.

[6] P. Devarajan, "Neutrophil gelatinase-associated lipocalin (NGAL): a new marker of kidney disease," Scandinavian Journal of Clinical and Laboratory Investigation, vol. 68, supplement 241, pp. 89-94, 2008.

[7] P. Devarajan, J. Mishra, S. Supavekin, L. T. Patterson, and S. S. Potter, "Gene expression in early ischemic renal injury: clues towards pathogenesis, biomarker discovery, and novel therapeutics," Molecular Genetics and Metabolism, vol. 80, no. 4, pp. 365-376, 2003.

[8] K. M. Schmidt-Ott, K. Mori, Y. L. Jau et al., "Dual action of neutrophil gelatinase-associated lipocalin," Journal of the American Society of Nephrology, vol. 18, no. 2, pp. 407-413, 2007.

[9] M. Haase, R. Bellomo, P. Devarajan, P. Schlattmann, and A. Haase-Fielitz, "Accuracy of neutrophil gelatinase-associated lipocalin (NGAL) in diagnosis and prognosis in acute kidney injury: a systematic review and meta-analysis," American Journal of Kidney Diseases, vol. 54, no. 6, pp. 1012-1024, 2009.

[10] J. L. Koyner, M. R. Bennett, E. M. Worcester et al., "Urinary cystatin $\mathrm{C}$ as an early biomarker of acute kidney injury following adult cardiothoracic surgery," Kidney International, vol. 74, no. 8, pp. 1059-1069, 2008.

[11] J. Mishra, C. Dent, R. Tarabishi et al., "Neutrophil gelatinaseassociated lipocalin (NGAL) as a biomarker for acute renal injury after cardiac surgery," The Lancet, vol. 365, no. 9466, pp. 1231-1238, 2005.

[12] V. S. Vaidya, S. S. Waikar, M. A. Ferguson et al., "Urinary biomarkers for sensitive and specific detection of acute kidney injury in humans," Clinical and Translational Science, vol. 1, no. 3, pp. 200-208, 2008.

[13] R. Bellomo, C. Ronco, J. A. Kellum, R. L. Mehta, and P. Palevsky, "Acute renal failure-definition, outcome measures, animal models, fluid therapy and information technology needs: the second international consensus conference of the acute dialysis quality initiative (ADQI) group," Critical Care, vol. 8, no. 4, pp. R204-212, 2004.

[14] A. A. N. M. Royakkers, J. C. Korevaar, J. D. E. Van Suijlen et al., "Serum and urine cystatin $\mathrm{C}$ are poor biomarkers for acute kidney injury and renal replacement therapy," Intensive Care Medicine, vol. 37, no. 3, pp. 493-501, 2011.

[15] W. A. Knaus, E. A. Draper, D. P. Wagner, and J. E. Zimmerman, "APACHE II: a severity of disease classification system," Critical Care Medicine, vol. 13, no. 10, pp. 818-829, 1985.

[16] J. R. Le Gall, S. Lemeshow, and F. Saulnier, "A new simplified acute physiology score (SAPS II) based on a European/North American multicenter study," Journal of the American Medical Association, vol. 270, no. 24, pp. 2957-2963, 1993.

[17] A. S. Levey, J. P. Bosch, J. B. Lewis, T. Greene, N. Rogers, and D. Roth, "A more accurate method to estimate glomerular filtration rate from serum creatinine: a new prediction equation," Annals of Internal Medicine, vol. 130, no. 6, pp. 461470, 1999.

[18] B. Z. Morgenstern, L. Butani, P. Wollan, D. M. Wilson, and T. S. Larson, "Validity of protein-osmolality versus proteincreatinine ratios in the estimation of quantitative proteinuria from random samples of urine in children," American Journal of Kidney Diseases, vol. 41, no. 4, pp. 760-766, 2003.

[19] S. M. Bagshaw, M. Bennett, M. Haase et al., "Plasma and urine neutrophil gelatinase-associated lipocalin in septic versus nonseptic acute kidney injury in critical illness," Intensive Care Medicine, vol. 36, no. 3, pp. 452-461, 2010.

[20] E. D. Siew, M. E. Matheny, T. A. Ikizler et al., "Commonly used surrogates for baseline renal function affect the classification 
and prognosis of acute kidney injury," Kidney International, vol. 77, no. 6, pp. 536-542, 2010.

[21] A. Prabhu, D. I. Sujatha, B. Ninan, and M. A. Vijayalakshmi, "Neutrophil gelatinase associated lipocalin as a biomarker for acute kidney injury in patients undergoing coronary artery bypass grafting with cardiopulmonary bypass," Annals of Vascular Surgery, vol. 24, no. 4, pp. 525-531, 2010.

[22] G. Wagener, M. Jan, M. Kim et al., "Association between increases in urinary neutrophil gelatinase-associated lipocalin and acute renal dysfunction after adult cardiac surgery," Anesthesiology, vol. 105, no. 3, pp. 485-491, 2006.

[23] C. L. Dent, Q. Ma, S. Dastrala et al., "Plasma neutrophil gelatinase-associated lipocalin predicts acute kidney injury, morbidity and mortality after pediatric cardiac surgery: a prospective uncontrolled cohort study," Critical Care, vol. 11, no. 6, p. R127, 2007.

[24] C. Xin, X. Yulong, C. Yu, C. Changchun, Z. Feng, and M. Xinwei, "Urine neutrophil gelatinase-associated lipocalin and interleukin-18 predict acute kidney injury after cardiac surgery," Renal Failure, vol. 30, no. 9, pp. 904-913, 2008.

[25] M. Bennett, C. L. Dent, Q. Ma et al., "Urine NGAL predicts severity of acute kidney injury after cardiac surgery: a prospective study," Clinical Journal of the American Society of Nephrology, vol. 3, no. 3, pp. 665-673, 2008.

[26] F. Capuano, M. Goracci, R. Luciani et al., "Neutrophil gelatinase-associated lipocalin levels after use of mini-cardiopulmonary bypass system," Interactive Cardiovascular and Thoracic Surgery, vol. 9, no. 5, pp. 797-801, 2009.

[27] S. M. Tuladhar, V. O. Püntmann, M. Soni, P. P. Punjabi, and R. G. Bogle, "Rapid detection of acute kidney injury by plasma and urinary neutrophil gelatinase-associated lipocalin after cardiopulmonary bypass," Journal of Cardiovascular Pharmacology, vol. 53, no. 3, pp. 261-266, 2009.

[28] K. Makris, N. Markou, E. Evodia et al., "Urinary neutrophil gelatinase-associated lipocalin (NGAL) as an early marker of acute kidney injury in critically ill multiple trauma patients," Clinical Chemistry and Laboratory Medicine, vol. 47, no. 1, pp. 79-82, 2009.

[29] M. Zappitelli, K. K. Washburn, A. A. Arikan et al., "Urine neutrophil gelatinase-associated lipocalin is an early marker of acute kidney injury in critically ill children: a prospective cohort study," Critical Care, vol. 11, p. R84, 2007.

[30] D. S. Wheeler, P. Devarajan, Q. Ma et al., "Serum neutrophil gelatinase-associated lipocalin (NGAL) as a marker of acute kidney injury in critically ill children with septic shock," Critical Care Medicine, vol. 36, no. 4, pp. 1297-1303, 2008.

[31] D. N. Cruz, M. De Cal, F. Garzotto et al., "Plasma neutrophil gelatinase-associated lipocalin is an early biomarker for acute kidney injury in an adult ICU population," Intensive Care Medicine, vol. 36, no. 3, pp. 444-451, 2010.

[32] J. M. Constantin, E. Futier, S. Perbet et al., "Plasma neutrophil gelatinase-associated lipocalin is an early marker of acute kidney injury in adult critically ill patients: a prospective study," Journal of Critical Care, vol. 25, no. 1, pp. 176-e1, 2010.

[33] H. R. H. de Geus, J. Bakker, E. M. E. H. Lesaffre, and J. L. M. L. Le Noble, "Neutrophil gelatinase-associated lipocalin at ICU admission predicts for acute kidney injury in adult patients," American Journal of Respiratory and Critical Care Medicine, vol. 183, no. 7, pp. 907-914, 2011.

[34] J. Mårtensson, M. Bell, A. Oldner, S. Xu, P. Venge, and C. R. Martling, "Neutrophil gelatinase-associated lipocalin in adult septic patients with and without acute kidney injury," Intensive Care Medicine, vol. 36, no. 8, pp. 1333-1340, 2010.

[35] K. Mori, H. T. Lee, D. Rapoport et al., "Endocytic delivery of lipocalin-siderophore-iron complex rescues the kidney from ischemia-reperfusion injury," Journal of Clinical Investigation, vol. 115, no. 3, pp. 610-621, 2005. 


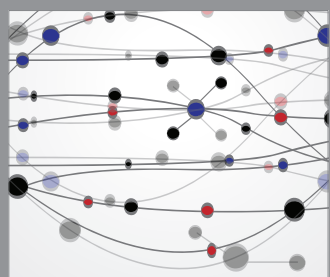

The Scientific World Journal
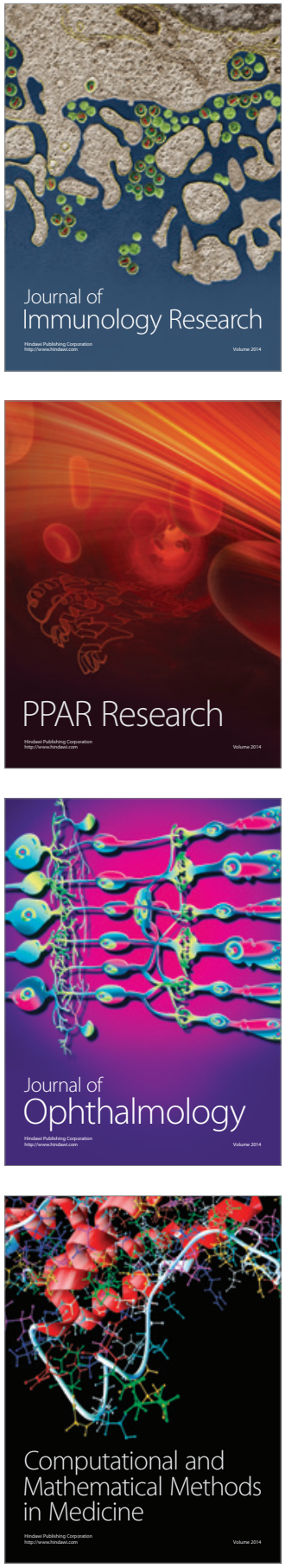

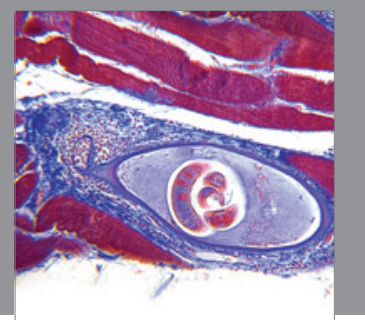

Gastroenterology

Research and Practice
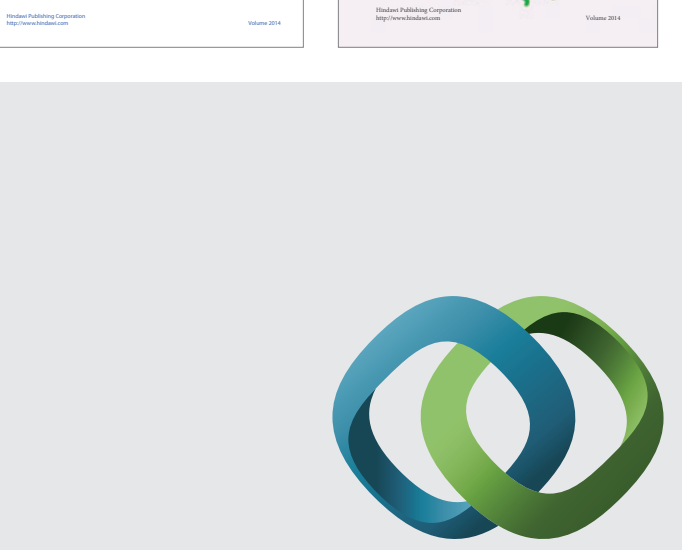

\section{Hindawi}

Submit your manuscripts at

http://www.hindawi.com
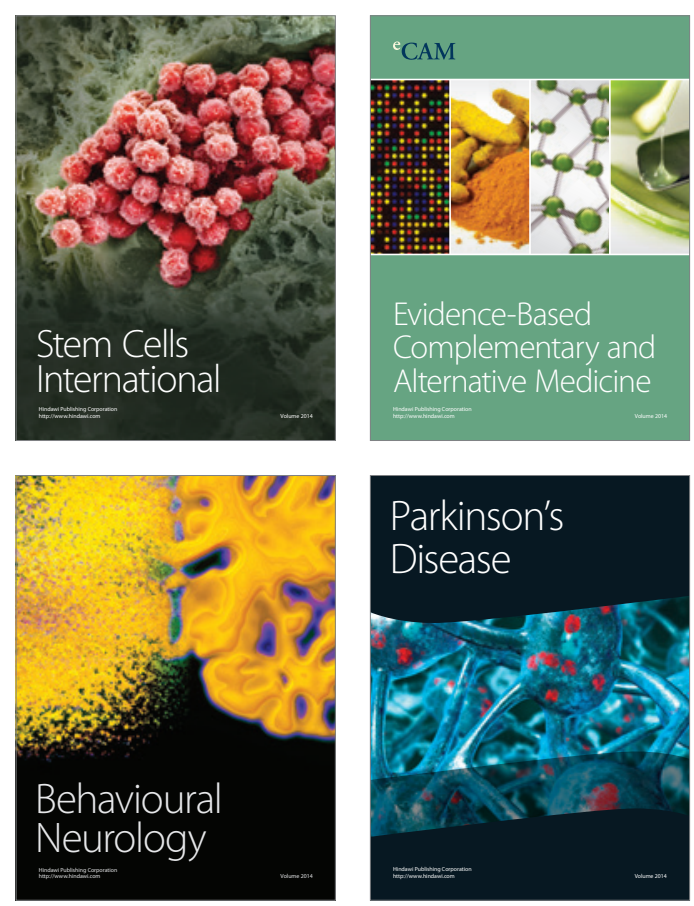

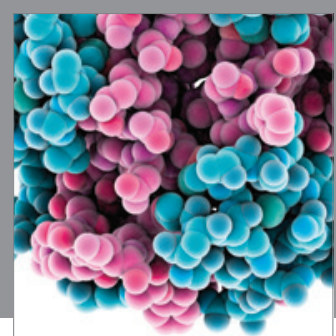

Journal of
Diabetes Research

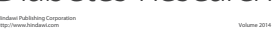

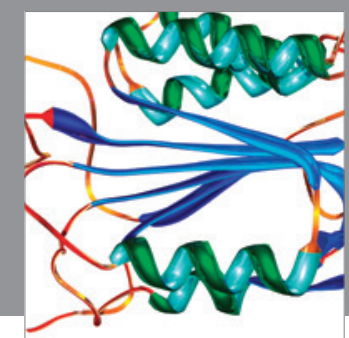

Disease Markers
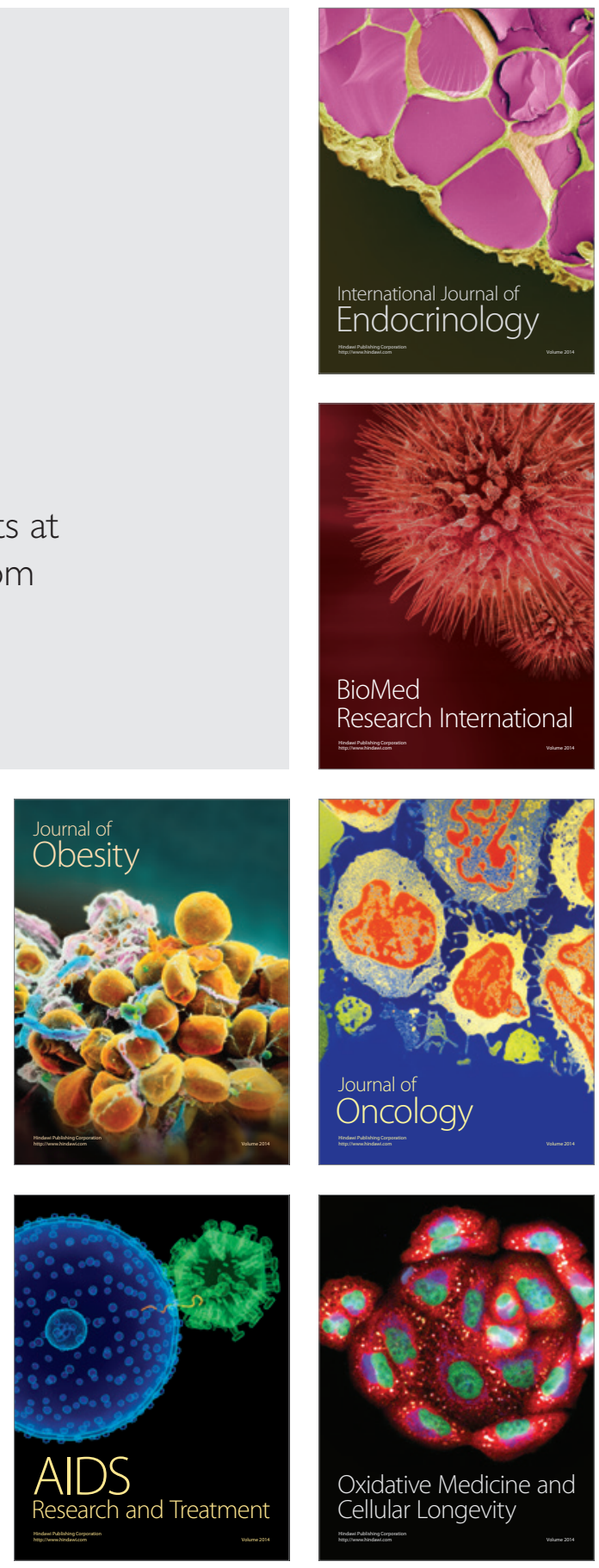